\title{
Isolated gross haematuria: A rare clinical presentation in neonate with urinary tract infection
}

\author{
Kayastha P', Basnet R², Adhikari RK ${ }^{3}$ \\ ${ }^{1}$ Pawana Kayastha, Lecturer; ${ }^{2}$ Rydam Basnet, Resident; ${ }^{3}$ Ramesh Kant Adhikari, Professor; Deapartment of Paeditrics, \\ Kathmandu Medical College Teaching Hospital, Kathmandu, Nepal
}

\begin{abstract}
Gross haematuria is uncommon in the first month of life and etiologies of haematuria in the newborn is different from those in older children. we reported a six days old term male baby who was brought by parents with isolated complain of red colored urine for one day and his mother had culture positive urinary tract infection prior to delivery. The baby was diagnosed to have culture positive urinary tract infection. Our findings suggest the diversity of clinical presentation of urinary tract infection in neonates and also some relationship between the history of urinary tract infection in mother and occurrence of urinary tract infection in neonate
\end{abstract}

Key words: Haematuria, Neonate, Urinary tract infection

\section{INTRODUCTION}

T Taematuria in newborn is uncommon'. Studies - Lon incidence of haematuria in infants is scarce. A retrospective study of infants with gross haematuria over a 17-year period between 1950 and 1967 estimated an incidence rate of 0.21 per 1000 admissions in infants younger than one month ${ }^{2}$. Haematuria when present has a wide differential diagnosis including renal vein thrombosis, polycystic kidney disease, obstructive nephropathy, tumor, congenital malformations, urinary tract infection (UTI), and acute kidney injury33.

The clinical manifestations of UTI in newborn infants are extremely variable, ranging from severe illness to nonspecific signs and symptoms, such as growth failure, vomiting, diarrhea, fever, irritability, lethargy, abnormal urination- namely oliguria, polyuria or malodorous urine, and jaundice ${ }^{4}$. This infection is an unusual occurrence during the first three days after birth ${ }^{5}$. Here we report the earliest case of UTI described in term uncomplicated newborn infant. The unusual features of this case included early gross haematuria as the only presenting symptom. Reports

Address for correspondence

Dr. Pawana Kayastha

Lecturer, Department Of Paediatrics

Kathmandu Medical College Teaching Hospital

Sinamangal, Kathmandu, Nepal

E-mail: paw_ana@hotmail.com of urinary analysis, urine cultures and abdominal ultrasonography established the diagnosis of UTI.

\section{CASE REPORT}

A six day old male neonate presented at Paediatrics outpatient departmentwith complain of passage of red coloured urine, multiple episodes for one day. The neonate was exclusivly breastfed, feeding well and was passing stool normally.There was no history of any injury at genitalia. or drug intake, fever. He was born at 39 weeks of gestation via emergency caesarean section (CS) for meconium stained amniotic fluid. APGAR scores was 7/10, 8/10 at one and five minutes respectively . His birth weight was 3350 grams. He received vitamin $\mathrm{K}$ one mg intramuscular at birth. There was no history of any umbilical artery catheterisation, sepsis, shock at birth and he was discharged as well baby after 24 hours of observation. In maternal history, the mother had UTI at third trimester of pregnancy detected just before delivery and treatment started thereafter. The mother's urine culture isolated Escherichia coli (gram negative organism) which was sensitive to ceftriaxone, cotrimoxazole, ofloxacin, norfloxacin, amikacin and nitrofurantoin. There was no history of bleeding problem in family. On physical examination of the neonate, no abnormalities were noticed and his vitals were also normal. Systemic examination was unremarkable. 
Investigation after admission revealed plenty of red blood cells/hpf, pus cells 4-6/hpf, epithelial cells 2-4/ hpf in urine analysis. Bleeding time, clotting Time, prothrombin Time, activated partial thromboplastin time were within normal limits for the age. Renal function tests as well as abdominal ultrasonography revealed normal findings. His hemoglobin was 17.8 $\mathrm{gm} / \mathrm{dl}$ and total and differential leucocyte counts were normal for the age. Platelet count was also normal. C-reactive protein was positive. Urine culture isolated Klebsiella species which was sensitive to ceftriaxone, ofloxacin, norfloxacin, amikacin. He was treated with intravenous cefotaxime and amikacin initially and oral ofloxacin was added after sensitivity pattern when culture report arrived which was continued for a total of 10 days. Haematuria resolved two days after antibiotic and urine was also normal.

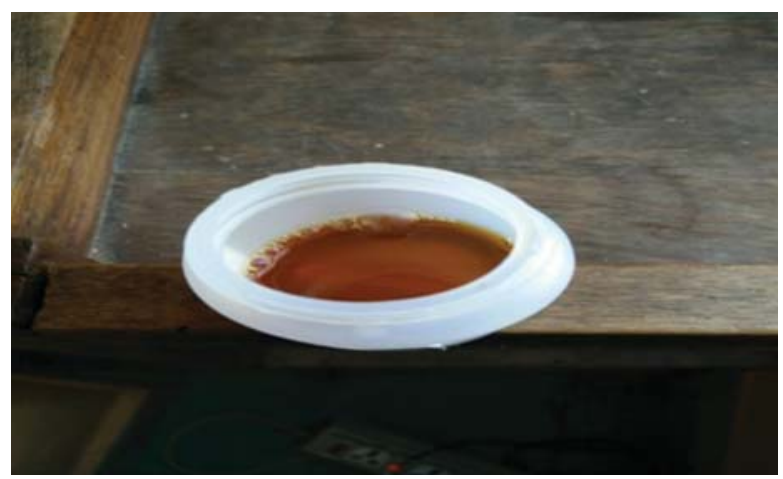

Figure 1:Urine sample of the newborn showing haematuria.

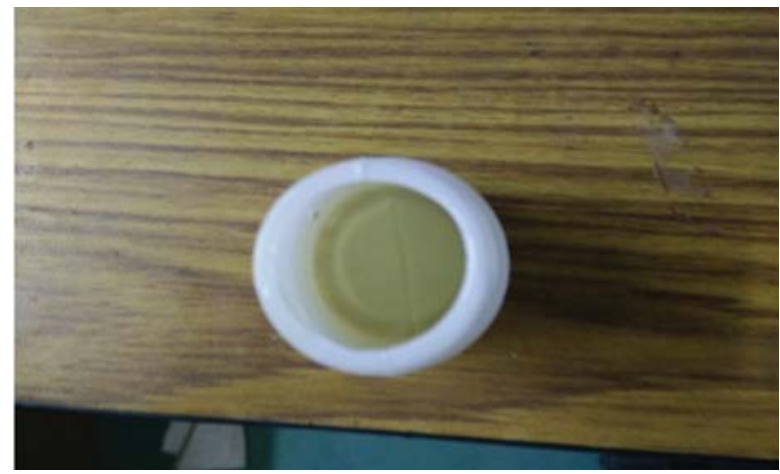

Figure 2: Urine sample of the newborn after treatment with antibiotic.

\section{DISCUSSION}

Prevalence of UTI is 0.1 percent to one percent in term neonates, predominantly seen in males with male: female ratio of 2:1). However, it is considerably more common in very low weight infants with prevalence between four percent and $25 \%{ }^{4}$. Common presenting features of UTI in neonates are irritability $(60 \%)$, poor feeding (50\%), and vomiting or diarrhea (up to $40 \%)^{6}$. Other findings are fever (20 to 40 percent), failure to thrive (15 to 43 percent), jaundice ( 3 to 41 percent), including abdominal distension resulting from ileus or enlarged kidneys caused by hydronephrosis ${ }^{5}$. The novelty of our case was the presence of only gross haematuria at six days of life. Similar case report was done by Verma et al at USA in 1998 where a three day old neonate had UTI who presented with early massive haematuria as the only presenting symptom and no risk factors for either sepsis or localization of infection in the genitourinary tract $^{7}$.

Haematuria at any age is abnormal and may indicate intrinsic renal damage or result from a bleeding or clotting abnormality. Moreover etiology of haematuria in the newbornis diverse, including renal vein or artery thrombosis, polycystic kidney disease, obstructive nephropathy, Wilms tumor, urological anamolies, urinary tract infection, and acute kidney injury. Hematuria may also be present in hemorrhagic disease of newbornand other severe bleeding abnormalities like disseminated intravascular coagulation, severe thrombocytopenia and clotting factors deficiency. Injury to urinary tract due to trauma or procedures like suprapubic bladder aspiration orurethral catheterization also leads to hematuria. The differential diagnosis for haematuria includes urate staining of the diaper, myoglobinuria, or hemoglobinuria. Vaginal bleeding in females or severe diaper rash is also possible cause of blood in diaper.

In our case most of the diseases were excluded by investigations carried out with urine, blood and certain radiological evaluation of the baby which did show normal values except for the presence of bacteria in urine hence diagnosing the disease as UTI. The organism isolated in this case was Klebsiella species (gram negative organism). Escherichia coli is the most prevalent cultured organism of neonatal urinary tract infections in many studies; however, other gram-negative bacilli such as Klebsiella species, Enterobacter, and Seratia speciesare also isolated ${ }^{4}$. Most UTIs in newborns affect upper tract rather than lower parts (simple cystitis) $)^{5}$. But in this case there was no features specifying upper tract involvement. In this case the maternal history revealed maternal UTI at third trimester of pregnancy detected just before delivery. Maternal UTI is independently associated with preterm delivery, preeclampsia, intrauterine growth retardation, and cesarean delivery. In addition, maternal UTI can be associated with some types of malformations as well. However, data regarding the association of maternal UTI with subsequent UTIs in the newborn babies are scarce. 
Neonatal UTI following untreated maternal UTI has been suggested as an important life-threatening event after delivery. In a recent study by Emamghorashi et al among Iranian neonates, a significant association was found between maternal UTI and neonatal UTI, while it revealed $30.0 \%$ of the neonates with UTI versus $6.8 \%$ of those without UTI had mothers with a history of UTI8. Emamghorashi et al has concluded that maternal UTI during pregnancy, especially in the third trimester was a risk factor for UTI in neonates.

In our case the mother's urine culture isolated E. coli (gram negative organism) which was sensitive to ceftriaxone, cotrimoxazole, ofloxacin, norfloxacin, amikacin and nitrofurantoin and mother was treated with nitrofurantoin after delivery.

Another study done by Khalesi et al has also shown a significant relationship between the occurrence of UTI in neonates and maternal UTI'. In the study, the trimester in which UTI had occurred were $4.4 \%, 6.1 \%$, and $4.4 \%$ during the first, second and third trimesters respectively. In his study the overall prevalence of UTI among neonates of affected mothers was significantly higher than that observed among non-infected mothers hence he concluded that maternal UTI results in 5.9 fold increased risk of neonatal UTI. However treatment modalities in mother for UTI were not mentioned. So possibly our case was the neonatal outcome of maternal UTI as no other risk factor for either sepsis or localization of infection in the genitourinary tract

\section{REFERENCES}

1. Askenazi DJ, Goldstein SL. Renal conditions. In: Cloherty JP, Eichenwald EC, Stark AR, editors. Manual of neonatal care.7th edn. Philadelphia: LWW; 2012: 350-76p.

2. Jernigan SM. Hematuria in theNewborn. ClinPerinatol. 2014 Sep;41(3):591-60.

3. Bates CM, Schwaderer AL. Clinical Evaluation of Renal and Urinary Tract Disease. In: Gleason CA, Devaskar SU eds. Avery's Diseases of Newborn. 9th edn. Philadelphia, Saunders 2012:1179p.

4. Alizadeh TP, Navabi B, Shariat M.Neonatal urinary tract infection: clinical response to empirical therapy versus in vitro susceptibility at Bahrami Children's Hospital-Neonatal Ward: 2001-2010. ActaMedicalranica. 2012;50(5): 348-52. was detected. Urinary tract infection poses the risk of mortality and morbidity in neonates. Various risk factors such as abnormal urinary tract are associated with UTI in newborns. A haematogenous spread of infection has been thought to be responsible for neonatal UTI because upper tract infection with associated bacteraemia is common. However, the microbiology of these infections and the high incidence of urinary tract abnormalities raise the question of whether neonatal UTI truly arises from hematogenous spread from a remote source or, in the majority of cases, actually represent an ascending urinary tract infection with an associated bacteremia.

E. coli is the most common uropathogen among neonates with UTI'. E coli colonies can be extensively shared among human and animal household members in the absence of sexual contact and in patterns suggesting host-to-host transmission ${ }^{8}$. Within household sharing of uropathogen organisms may also be involved in neonatal UTI. A higher prevalence of UTI in neonates of mother with UTI may be the result of such uropathogen sharing.

\section{CONCLUSION}

Isolated haematuria in a neonate is rare and should always be investigated thoroughly. It is not clear whether neonatal UTI occurs secondary to bacteremia or ascending urinary tract infection or associated with maternal UTI. This case however may indicate a possible benefit of evaluation of neonates of mothers who had UTI during pregnancy.

5. Ashkan M. Urinary tract infections in newborns. Pediatric on call Journal. 2008 April:5(4):1-10.

6. Zderic SA. Urinary Tract Infections and Vesicoureteral Reflux. In: Gleason CA, Devaskar SU eds. Avery's Diseases of Newborn. 9th edn. Philadelphia, Saunders 2012:1228p.

7. Verma RP, Pizzica A. Early neonatal urinary tract infection: a case report and review. J Perinatol. 1998 Nov-Dec;18(6):480-4.

8. Emamghorashi F, Mahmoodi N, Tagarod Z, HeydariST.Maternal Urinary Tract Infection as a Risk Factor For Neonatal Urinary Tract Infection. IJKD. 2012 May;6:178-80

9. Khalesi N, Khosravi N, Jalali A, Amini L. Evaluation of Maternal Urinary Tract Infection as a Potential Risk Factor for Neonatal Urinary Tract Infection. J Family Reprod Health. 2014 Jun;8(2):59-62. 